\title{
Nodular Distribution Pattern on Chest Computed Tomography in Patients Diagnosed with Nontuberculous Mycobacteria Infection
}

Marušić Ante, Department of Radiology, Thoracic radiology, University Hospital Center, University of Zagreb Medical School, Kišpatićeva, Croatia

Kuhtić Ivana, Department of Radiology, Thoracic radiology, University Hospital Center, University of Zagreb Medical School, Kišpatićeva 12, 10000 Zagreb, Croatia, email: ikuhtic@gmail.com, Contact number: 00385989398206 - Corresponding author

Mažuranić Ivica, Department of Radiology, Thoracic radiology, University Hospital Center, University of Zagreb Medical School, Kišpatićeva, Croatia

Janković Mateja, Department for Respiratory Diseases, University Hospital Center, University of Zagreb Medical School, Kišpatićeva, Croatia

Glodić Goran, Department for Respiratory Diseases, University Hospital Center, University of Zagreb Medical School, Kišpatićeva, Croatia

Sabol Ivan, Division of Molecular medicine, Laboratory of Molecular Virology and Bacteriology, Ruder Boskovic Institute, Bijenička cesta, Croatia

Stanić Lucija, Emergency Department of Zagreb County, Matice Hrvatske, Croatia 
Conflict of interest: All authors declare that they have no conflict of interest.

Ethical statement: All procedures performed in this study involving human participants were in accordance with the ethical standards of the institutional and/or national research committee and with the 1964 Helsinki Declaration and its later amendments or comparable ethical standards. For this type of study, formal consent is not required.

Informed consent: This is a retrospective study. For this type of study, formal consent is not required, because no personal data was contained and there is no concern about identifying information.

Author Contribution: All authors contributed to the study conception and design. All authors commented on previous versions of the manuscript. All authors read and approved the final manuscript.

Funding and acknowledgments: None. 


\begin{abstract}
Purpose: This study aims to evaluate the prevalence of spreading pathways in Nontuberculous Mycobacteria (NTM) pulmonary infections according to nodular distribution patterns seen on Chest Computed Tomography (CT).
\end{abstract}

Materials and Methods: This study included 63 patients diagnosed with NTM lung infections who underwent $\mathrm{CT}$ at our institution. A retrospective analysis of CT images focused on the presence of nodules and their distribution, presence of intrathoracic lymphadenopathy and the predominant side of infection in the lung. The findings were classified into five groups; centrilobular (bronchogenic spread), perilymphatic (lymphangitic spread), random (haematogenous spread), combined pattern and no nodules present. The groups were then mutually compared according to other CT findings.

Results: Among 51 (81\%) patients identified with nodular pattern on chest CT, 25 (39.8\%) presented with centrilobular, 7 (11.1\%) with perilymphatic, 6 (9.5\%) with random, and $13(20.6 \%)$ with combined nodular patterns but located in different area in the lung. The predominance of the right side of the lung was detected in 38 cases (60.3\%). Intrathoracic lymphadenopathy was evident in 20 patients $(31.7 \%)$. Significant differences in distributions of chest CT nodular patterns were seen in patients infected with Mycoplasma avium complex (MAC) associated with centrilobular pattern $(p=0.0019)$ and $M$. fortuitum associated with random pattern $(p=0.0004)$. Some of the findings were related to perilymphatic nodules between other isolated species of NTM $(\mathrm{p}=0.0379)$, intrathoracic lymphadenopathy $(\mathrm{p}=0.0285)$ and the predominance of the upper right lobe $(\mathrm{p}<0.0001)$.

Conclusion: The results of this study showed a high proportion of perilymphatic nodules and right-sided predominance in the upper lobe, which, combined with intrathoracic lymphadenopathy is highly suggestive of the lymphangitic spread of lung NTM infections based on imaging alone.

Keywords: Multidetector Computed Tomography, Multiple Pulmonary Nodules, Nontuberculous Mycobacteria, Lymph 


\section{Abbreviations:}

NTM - Nontuberculous Mycobacteria

TB - Tuberculosis

M. - Mycobacteria

CT - Computed Tomography

HRCT - High-Resolution Computed Tomography

MAC - Mycobacterium Avium Complex 


\title{
Nodular Distribution Pattern on Chest Computed Tomography in Patients Diagnosed with Nontuberculous Mycobacteria Infection
}

\begin{abstract}
Purpose: This study aims to evaluate the prevalence of spreading pathways in Nontuberculous Mycobacteria (NTM) pulmonary infections according to nodular distribution patterns seen on Chest Computed Tomography (CT).

Materials and Methods: This study included 63 patients diagnosed with NTM lung infections who underwent CT at our institution. A retrospective analysis of CT images focused on the presence of nodules and their distribution, presence of intrathoracic lymphadenopathy and the predominant side of infection in the lung. The findings were classified into five groups; centrilobular (bronchogenic spread), perilymphatic (lymphangitic spread), random (haematogenous spread), combined pattern and no nodules present. The groups were then mutually compared according to other CT findings.
\end{abstract}

Results: Among 51 (81\%) patients identified with nodular pattern on chest CT, 25 (39.8\%) presented with centrilobular, $7(11.1 \%)$ with perilymphatic, $6(9.5 \%)$ with random, and $13(20.6 \%)$ with combined nodular patterns but located in different area in the lung. The predominance of the right side of the lung was detected in 38 cases $(60.3 \%)$. Intrathoracic lymphadenopathy was evident in 20 patients $(31.7 \%)$. Significant differences in distributions of chest CT nodular patterns were seen in patients infected with Mycoplasma avium complex (MAC) associated with centrilobular pattern $(\mathrm{p}=0.0019)$ and $M$. fortuitum associated with random pattern $(\mathrm{p}=0.0004)$. Some of the findings were related to perilymphatic nodules between other isolated species of NTM $(p=0.0379)$, intrathoracic lymphadenopathy $(p=0.0285)$ and the predominance of the upper right lobe $(\mathrm{p}<0.0001)$

Conclusion: The results of this study showed a high proportion of perilymphatic nodules and rightsided predominance in the upper lobe, which, combined with intrathoracic lymphadenopathy is highly suggestive of the lymphangitic spread of lung NTM infections based on imaging alone.

Keywords: Multidetector Computed Tomography, Multiple Pulmonary Nodules, Nontuberculous Mycobacteria, Lymph 
Abbreviations: NTM - Nontuberculous Mycobacteria, TB - Tuberculosis, M. - Mycobacteria, CT - Computed Tomography, HRCT - High-Resolution Computed Tomography, MAC - Mycobacterium Avium Complex 


\section{INTRODUCTION}

The well-known nodular patterns that can be detected on high-resolution computed tomography (HRCT) of the lung can be classified as centrilobular, perilymphatic and random. The different nodular patterns are shown to correlate with spread patterns as bronchogenic, lymphangitic and hae matogenous spread, respectively [1-3].

Centrilobular distribution of the nodular pattern is most often caused by bronchiolar diseases that show centrilobular nodules that are separated from the pleural surfaces and interlobular septa by a distance of 5-10 $\mathrm{mm}$ with no nodules present in the subpleural spaces or other compartments where lymph vessels are located. Centrilobular nodular opacities can be associated with the presence of intralobular branching lines, creating the tree-in-bud pattern [4]. The presence of tree-in-bud is indicative of small airway disease and is found in the majority of cases associated with airway infection. While centrilobular nodular opacities are seen in lymphatic and perilymphatic diseases, nodular opacities are commonly seen in other lung compartments containing lymphatic vessels (interlobular septa, subpleural space and axial peribronchovascular connective tissue) as well. However when nodules are separated from the pleural surfaces and the interlobular septa by a distance of 5-10 $\mathrm{mm}$ and no nodules in the subpleural spaces or other compartments where lymph vessels are visualized, a centrilobular distribution of the nodular pattern caused by either bronchiolar/peribronchiolar disease or vascular/perivascular disease can be concluded [5].

A nodular pattern with perilymphatic distribution typically shows peribronchovascular nodules, subpleural nodules, nodules in the interlobular septa and centrilobular nodules according to the anatomy of the lung and secondary pulmonary lobule [6]. Since no lymphatics are present distal to the respiratory bronchioles, no nodules are seen between the core of the secondary pulmonary lobule and the interlobular septa.

Random distribution refers to the random location of the nodules in relation to the structures of the secondary pulmonary lobule. Nodules may be located near the centre of a lobule, near the interlobular septa and in the lung parenchyma in between. They can also be seen in relation to the pleural surfaces and the pulmonary arterial branches (the so-called feeding vessels) but have no consistent or predominant relationship with any of these structures or the structures of the secondary pulmonary lobule. Random distribution of nodules results from a vascular spread of disease, in which the pathological agent may pass the centrilobular area and distribute along the small vessels and capillaries of the lobule [7]. 
Nontuberculous mycobacteria (NTM) infections usually present with consolidation with or without cavitation, centrilobular nodules/bronchiolitis and bronchiectasis without significant lymphadenopathy [8-13].

To our knowledge, limited data have been published on nodular patterns detectable on chest CT regarding spread pathways in NTM lung infections.

There are, however, relevant studies on Tuberculosis (TB) spread patterns within the lung. Recent studies, namely Ko et al. [14], show a relevant proportion of lymphangitic spread in the lung among TB patients, atypical CT manifestations of Tuberculosis infection with characteristic pathomorphological appearances for lymphangitic spread consistent with perilymphatic nodular pattern and a predominance of right and upper part of the lung and intrathoracic lymphadenopathy [14-17].

The other two patterns of TB lung infection spread, bronchogenic (bronchiolitis) and haematogenous (miliary TB), are well known [18-22].

Many studies on NTM lung infection spread focus on a local/continuous spread in view of consolidation with or without cavitation [4], bronchogenic spread - centrilobular peribronchial nodules ("tree-in-bud" pattern) [23-24] and haematogenous spread - random nodular pattern [25]. Other studies compare the radiomorphological presentation of TB and NTM lung infections, showing similar patterns, including both consolidation and nodular appearances, but to a lesser extent [26-28]. It has not been shown that there is any significant difference in CT features of lung infection between different NTM species in previous studies.

Considering the above-mentioned TB and NTM lung infection similarities, and lack of in-depth approach to spread patterns of NTM infections on imaging, particularly lymphangitic spread, it was important to investigate this further. Since established nodular patterns can be correlated to spread pathways of infection, this could be the best way to learn more about NTM biology from their CT presentation.

Therefore, the aim of this study was to assess the frequency and distribution of lung nodules detectable on chest CT in patients with NTM isolates to confirm different infection spread pathways in the lung based on imaging characteristics alone.

\section{MATERIALS AND METHODS}


All procedures performed in this study involving human participants were in accordance with the ethical standards of the institutional and/or national research committee and with the 1964 Helsinki Declaration and its later amendments or comparable ethical standards. For this type of study, formal consent is not required, because the data were viewed retrospectively and no personal data was contained in this study.

The study was based on institutional data for NTM infections over two years (2016 and 2017). The NTM infection diagnosis was established locally and the culture confirmed by the National Mycobacteria Reference Laboratory of the Croatian National Institute of Public Health (CNIPH), Zagreb. Cultures were obtained via bronchoalveolar lavage (BAL), aspiration from bronchi with the catheter and/or in sputum with PCR/DNA based methods. A total of 131 persons with positive NTM culture were identified. Fifty-six of them did not have available CT scan and therefore were excluded from the study. Patients with disseminated malignant disease $(n=7)$, lymphoproliferative disorders $(n=3)$, and established sarcoid $(n=2)$ were also excluded from the study. There were no patients with HIV infection or other immunosuppressive disorders.

Finally, the study included 63 patients, all of which had CT done around the time of diagnosis in our institution (within a month). As there was no CT protocol in place for NTM infections, different protocols for CT scanning were used: usual chest CT with or without IV administration of contrast, CT pulmonary angiogram and HRCT. There were also multiple CT scanners involved (Siemens Healthcare and General Electric devices). Therefore, scanning parameters varied. The slice thickness was 1 or $1.25 \mathrm{~mm}$. The images were analysed on standard PACS workstations.

All CT examinations included in the study were double read by 2 consultant radiologists with special interest and experience in thoracic radiology (11 and 25 years). The readers worked independently of each other, aware of NTM infection present. All disagreements were resolved in the discussion, resulting in the agreement of both readers. The agreement level between the two radiologists for the presence or absence of each CT finding was tested using kappa statistics. The interobserver agreement between the two radiologists for identifying nodules and predominance of the affected side of the lung was almost perfect $(0.8<\mathrm{K}<0.92)$. There was a substantial agreement in identifying the perilymphatic, centrilobular and random distribution of nodules and for identifying intrathoracic lymphadenopathy $(0.68<\mathrm{K}<0.79)$.

The findings were classified according to CT appearances of nodules, intrathoracic lymphadenopathy and predominant part of the affected lung, focusing particularly on nodules. Considering different nodular 
patterns, findings were further divided into five groups (centrilobular, random, perilymphatic, combined patterns and no nodules). The groups were then mutually compared according to other CT findings.

The distribution was tested against equal frequency assumptions. Comparison between groups was performed using the independent t-test for continuous variables and the chi-square or Fisher exact test for categorical variables. Fisher $2 \times 5$ exact test was used to compare different nodular patterns in when more than $2 \times 2$ groups were compared [29]. For dichotomous outcomes, the binomial test was used. In all tests, $p<0.05$ was considered statistically significant.

\section{RESULTS}

The radiologic findings in 63 patients diagnosed with a pulmonary infection caused by NTM were analysed (40 men and 23 women; mean age 63 years; age range $11-85$ years).

\subsection{Nodules}

For this study, only nodular patterns of lung infection were assessed in detail. Of 63 patients included in the study, 51 (81\%) were detected with nodules. The centrilobular nodular pattern was detected on 25 (39.8\%), perilymphatic on $7(11.1 \%)$, and random on 6 occasions $(9.5 \%)$.

There were 13 (20.6\%) patients with combined nodular patterns. In three patients all nodular patterns were detected at the same time point, but confined to smaller areas in different lung regions. Furthermore, ten patients had been detected with two synchronous nodular patterns in different combinations: centrilobular with perilymphatic $(\mathrm{n}=5)$ and centrilobular with random $(\mathrm{n}=5)$. Interestingly, no patient had been identified with a combination of perilymphatic and random nodular distribution pattern.

\subsection{NTM species}

As per distribution of each nodular pattern concerning NTM species, there was a significant difference between Mycobacterium avium complex (MAC: M. avium and M. intracellulare), M. fortuitum and other NTM (M. abscessus, M. chimerae, M. malmoense, M. celatum, M. kansasii, M. segmatis) (Figure 1). 
In the MAC group $(n=20), 14$ cases of centrilobular-and a single case of random nodule pattern were detected. Interestingly, there were no cases of perilymphatic or combined nodule pattern in this group.

The distribution of nodular patterns was significantly different between MAC and non-MAC groups (Fishers $2 \times 5$ exact test, $\mathrm{p}=0.0006)$. The centrilobular pattern was the most responsible for the deviation (expected value 7.9, observed 14). This was further confirmed by comparing the distribution of the centrilobular pattern versus other patterns in MAC and non-MAC groups by Fishers exact $2 \times 2$ test $(\mathrm{p}=0.0019)$.

Similarly, in the NTM group without MAC $(n=43), 11$ cases of centrilobular, 7 perilymphatic and 5 random nodule distribution patterns were found. All of 7 perilymphatic and 15 combined nodule patterns were detected in non-MAC group while the expected values were 4.8 and 8.9 indicating these patterns also influence the overall significance of the distribution. Indeed, when comparing the distribution of combined pattern versus other patterns in MAC and non-MAC groups the distribution was significantly different (Fishers exact test 2x2, $\mathrm{p}=0.0057)$, while it did not reach statistical significance.

The distribution of different patterns in patients with $M$. fortuitum was also significantly different from those of patients without $M$. fortuitum (Fishers $2 \times 5$ exact test, $\mathrm{p}=0.0009$ ). The pattern of random nodules was detected in 5 cases of infections caused by $M$. fortuitum, while the expected value was 1.1 indicating that this finding was the most responsible for the observed deviation. This was confirmed by Fishers exact $2 \times 2$ comparison of the distribution of random pattern versus other patterns in $M$. fortuitum vs other groups $(p=0.0004)$.

The distributions of different patterns in patients infected with the more common $M$. xenopi $(\mathrm{n}=15)$ were not statistically different from patients without those NTM. However, the distribution of patterns in patients infected with all other NTM species combined as a group (M. abscessus, M. chimerae, M. malmoense, $M$. celatum, M. kansasii, M. segmatis) was borderline significantly different (Fishers $2 \times 5$ exact test, $\mathrm{p}=0.0455)$. The perilymphatic pattern was mainly responsible for this deviation as the expected value was 0.9 and 3 cases were observed. Comparing the perilymphatic vs other patterns in patients infected with other NTM group also indicated some statistical significance (Fisher $2 \times 2$ exact test, $\mathrm{p}=0.0379$ ).

\subsection{Intrathoracic lymphadenopathy}


Intrathoracic lymphadenopathy was found in 20 patients $(31.7 \%)$, mediastinal and hilar $(>10 \mathrm{~mm}$ in short-axis, axial plane).

There were no statistically significant differences in nodular patterns between patients with or without intrathoracic lymphadenopathy (Fishers $2 \times 5$ exact test, $\mathrm{p}=0.1307$ ). However, the highest proportion of intrathoracic lymphadenopathy was found in the group with a perilymphatic nodular pattern, in 5 of 7 cases (71.2\%). Comparing patients with perilymphatic pattern vs other patterns between patients with or without intrathoracic lymphadenopathy indicated that the difference might be significant (Fishers exact $2 \times 2, \mathrm{p}=0.0285$ ).

\subsection{Predominant part of the lung}

The majority ( $n=38,60.3 \%$ ) of patients had right lung predominance of lesions. Left lung predominance was shown in 13 cases (20.6\%). The upper lobes were affected in 33 patients $(52.4 \%)$ and lower lobes including the middle lobe in $18(28.6 \%)$.

Overall, the distribution of lung infection patterns were significantly different in patients where the right side was predominantly affected compared to patients where it was affected left side (Fishers $2 \times 5$ exact test, $\mathrm{p}<0.0001)$. The greatest deviation was due to centrilobular pattern, which was observed in 21 while being expected in 15.1 cases and due to perilymphatic pattern observed in 7 and expected in 4.2 cases. This was confirmed by comparing centrilobular vs other patterns in patients with or without right side predominance (Fishers $2 \times 2$ exact test, $\mathrm{p}=0.0033$; Figure 2).

The perilymphatic pattern was also significantly more often seen in patients with right side predominance compared to other patterns (Fishers $2 \times 2$ exact test, $\mathrm{p}=0.0358$ ). Similar results were seen when focusing on the upper right lobe (Fishers $2 \times 5$ exact test, $\mathrm{p}<0.0001$ ), while for lower right lobe the results were not significant (Fishers $2 \times 5$ exact test, $\mathrm{p}=0.4171$ ).

The distribution of patterns in patients with left lobe predominance was also significantly different when compared with other patients (Fishers $2 \times 5$ exact test, $p=0.0007$ ). The major source of deviation was the random pattern with an expected value of 1.2 but observed in 5 patients with left side predominance. When a random pattern was compared with other patterns in patients with or without left side predominance the distribution was also significantly different (Fishers exact test $2 \times 2, p=0.0009$ ). There were no significant differences when upper left lobe was further investigated, also differences in patterns of patients with lower left 
lobe predominance vs other patients approached but did not reach significance (Fishers exact test $2 \times 5$, $\mathrm{p}=0.0565)$.

Interestingly, in half of the patients with lower left lobe predominance a random nodular distribution pattern was seen (expected value 0.8 , observed value 3 ). Upon further investigation, the distributions of random vs non-random patterns in patients with or without lower left lobe involvement were significantly different (Fishers exact $2 \times 2$ test, $\mathrm{p}=0.0232$ ).

\subsection{In total}

Pulmonary infections caused by MAC are most commonly presented, except for consolidations and cavities, with a centrilobular nodule pattern with predominance in the right part of the lung and rarely with intrathoracic lymphadenopathy.

Interestingly, $M$. fortuitum infection is most commonly manifested by the random distribution of nodules with a tendency of localization in the left lower lobe of the lung and rarely with intrathoracic lymphadenopathy.

Non-MAC and other NTM infections are most commonly presented by perilymphatic and combined nodule patterns with predominance in the right upper lobe and intrathoracic lymphadenopathy (Figure 3).

\section{DISCUSSION}

Among presumed NTM infection spread pathways in the lung - bronchogenic, hematogenous and lymphangitic - the first two did not show significant divergence from already published data. However, this study showed results in keeping with lymphangitic dissemination of NTM lung infection; this appears not to have been described previously, although there are several studies on Mycobacterium tuberculosis (TB) that support the lymphangitic spread of TB lung infection. The lymphangitis in TB was described as early as 1873 by Klein [15], further assessed by Basaraba et al. [16], only to be correlated in detail for chest CT by Ko at al. [30] in 2015. Behr et al. recently explained TB pathogenesis using a lymphatic model based on considerable observational data from humans and natural or experimental animal models [17]. According to the lymphatic model, Mycobacterium tuberculosis invades the hilar nodes via lymphatics. In long standing lung TB infections, spread to lymph nodes can occur via pulmonary lymphatics. Further on, the infection can spread in a retrograde 
manner to sections of the lungs with lower vascular perfusion and difference in ventilation, with a right side and upper predominance, based on asymmetric lymphatic drainage [14]. This study is in line with limited previous data on the lymphangitic spread of TB.

Since mycobacterial infections, both TB and NTM, share most of the known biological and radiological features, we assumed that among other infection spread pathways, there should be lymphangitic spread as well.

The results of this study showed a significant correlation of perilymphatic nodules with right-sided predominance, in particular the upper lobe, and intrathoracic lymphadenopathy. The abnormalities mentioned above suggest the lymphangitic spread of NTM infections, which had been described for TB infections in previous studies $[15-17,30,31]$.

With the exclusion of MAC infections (which do not show CT signs of lymphangitic spread), other NTM infections show statistical significance in the occurrence of perilymphatic nodules ${ }_{2}$, the predominance of the right and upper part of the $\operatorname{lung}_{2}$ as well as a higher prevalence of intrathoracic lymphadenopathy. This supports a lymphangitic spread of NTM lung infection. In addition, the MAC infections, which again, do not show CT characteristics of lymphangitic spread $_{2}$ show statistical significance in the predominance of the right side of the lung, however, not in the predominance of the upper lobe and rarely with intrathoracic lymphadenopaty.

Not all NTM species in the current study showed the same spread pattern. For example, there was not a single MAC infection with a lymphangitic spread, while $M$. xenopi showed the most frequent lymphangitic spread pattern. This finding could be of value in assessing patients with COPD (Chronic Obstructive Pulmonary Disease), as chronic obstruction has been found more often in patients with M. xenopi infection [32]. Among random nodular distribution/haematogenous $\operatorname{spread}_{2}$ the most frequently isolated NTM was $M$. fortuitum. The above findings cannot be explained by available data, but the reason can lay in the different biological behaviour of NTM species due to genotype specificities.

It should also be noted that CT was performed within one month of diagnosis (in some cases even before, because the findings of a CT scan suggested a possible infection with NTM), but it is unknown how long the disease lasted before being diagnosed in cultures. The different types of NTM cause a different intensity of clinical symptoms, leading to a different period of time until diagnosis and a CT scan. In particular, MAC infections have more severe clinical symptoms and are diagnosed in the earlier stages of the disease. On the 
contrary, other NTM is diagnosed in the later stages of the disease because they cause milder clinical symptoms. This may also indicate a different chronological, pathomorphological presentation on CT scan that can indicate a lymphatic spread of the disease in the later phase of the disease. This is supported by a large number of cases with combined nodular patterns at different locations in the lung, as a possible transition phase.

The limitations of this study must be acknowledged. Unfortunately, the absolute number of different nodular patterns, including the highlighted perilymphatic nodules was often very small and this might affect statistical calculations. Certainly, the findings should be confirmed on a larger number of patients. Furthermore, we had no data about the pathohistological confirmation of chest CT findings.

In conclusion, lung nodules with perilymphatic distribution might be regarded as another CT feature of pulmonary infection caused by NTM. As already known, NTM lung infections can show local, bronchogenic and haematogenous spread as well. The awareness of NTM biology and lung infection spread pathways can help the radiologist to assess the spread and activity of disease with more accuracy and confidence. The importance of imaging is highlighted in the fact that morphological confirmation plays an important role in supporting clinical suspicion for NTM infection in reaching the final diagnoses.

Conflict of interest: All authors (Ante Marušić, Ivana Kuhtić, Ivica Mažuranić, Mateja Janković, Goran Glodić, Ivan Sabol, Lucija Stanić) declare that there is no conflict of interest.

Ethical statement: All procedures performed in this study involving human participants were in accordance with the ethical standards of the institutional and/or national research committee and with the 1964 Helsinki Declaration and its later amendments or comparable ethical standards.

Informed consent: This is a retrospective study. For this type of study, formal consent is not required, because no personal data was contained and there is no concern about identifying information.

Author Contribution: All authors contributed to the study conception and design. All authors commented on previous versions of the manuscript. All authors read and approved the final manuscript.

Funding and acknowledgments: None. 


\section{References:}

1. J. A. Verschakelen, W. De Wever Computed Tomography of the Lung, Springer Berlin Heidelberg New York, ISBN 978-3-642-39518-5

2. Remy-Jardin M, Beuscart R, Sault M, Marquette C, Remy J. Subpleural micronodules in diffuse infiltrative lung diseases: evaluation with thin section CT scans. Radiology 1990; 177:133-139

3. Raoof S, Amchentsev A, Vlahos I, Goud A, Naidich DP. Pictorial essay: multinodular disease-a highresolution CT scan diagnostic algorithm. Chest 2006; 129:805-815

4. Collins J, Blankenbaker D, Stern EJ. CT patterns of bronchiolar disease: what is tree-in-bud? AJR Am J Roentgenol 1998; 171:365-370

5. Gallardo X, Castaner E, Mata J et al. Nodular Pattern at Lung Computed Tomography in Fat Embolism Syndrome. A Helpful Finding. J Comput Assist Tomogr 2006; 30:254-257

6. Colby TV, Swensen SJ. Anatomic distribution and histopathologic patterns in diffuse lung disease: correlation with HRCT. J Thorac Imaging 1996; 11:1-26

7. Voloudaki AE, Tritou IN, Magkanas EG et al. HRCT in miliary lung disease. Acta Radiol 1999; $40: 451-456$

8. Kim TS, Koh WJ, Han J, Chung MJ, Lee JH, Lee KS, Kwon OJ. Hypothesis on the evolution of cavitary lesions in nontuberculous mycobacterial pulmonary infection: thin-section CT and histopathologic correlation. AJR Am J Roentgenol. 2005; 184(4):1247-52

9. McEvoy S, Lavelle L, Kilcoyne A, McCarthy C, deJong PA, Loeve M, Tiddens HA,McKone E, Gallagher CG, Dodd JD. High-resolution CT of nontuberculous mycobacterium infection in adult CF patients: diagnostic accuracy. Eur Radiol.2012 Dec;22(12):2736-42

10. Polverosi R, Guarise A, Balestro E, Carloni A, Dalpiaz G, Feragalli B. High-resolution CT of nontuberculous mycobacteria pulmonary infection in immunocompetent, non-HIV-positive patients. Radiol Med. 2010 Mar;115(2):191-204

11. Kwak N, Lee CH, Lee HJ, Kang YA, Lee JH, Han SK, Yim JJ. Non-tuberculous mycobacterial lung disease: diagnosis based on computed tomography of the chest. Eur Radiol. 2016 Dec;26(12):44494456 
12. Jeong YJ, Lee KS, Koh WJ, Han J, Kim TS, Kwon OJ. Nontuberculous mycobacterial pulmonary infection in immunocompetent patients: comparison of thin-section CT and histopathologic findings. Radiology. 2004 Jun;231(3):880-6

13. Lee KS, Kim TS, Han J, Hwang JH, Yoon JH, Kim Y, Yoo SY. Diffuse micronodular lung disease: HRCT and pathologic findings. J Comput Assist Tomogr. 1999 Jan-Feb;23(1):99-106

14. Ko JM, Park HJ, Cho DG, Kim CH. CT differentiation of tuberculous and non-tuberculous pleural infection, with emphasis on pulmonary changes. Int J Tuberc Lung Dis. 2015 Nov;19(11):1361-8

15. Klein E. Proceedings of the Royal Society of London. Proc R Soc London 1873; 22: 133-145

16. Basaraba RJ, Smith EE, Shanley CA, Orme IM. Pulmonary lymphatics are primary sites of Mycobacterium tuberculosis infection in guinea pigs infected by aerosol. Infect Immun. 2006 Sep;74(9):5397-401

17. Behr MA, Waters WR. Is tuberculosis a lymphatic disease with a pulmonary portal? Lancet Infect Dis. 2014 Mar;14(3):250-5

18. Pereira M, Gazzoni FF, Marchiori E, Irion K, Moreira J, Giacomelli IL, Pasqualotto A, Hochhegger B. High-resolution CT findings of pulmonary Mycobacterium tuberculosis infection in renal transplant recipients. Br J Radiol. 2016;89(1058):20150686

19. He W, Chen BD, Lv Y, Zhou Z, Xu JP, Lv PX, Zhou XH, Ning FG, Li CH, Wang DP, Zheng J. Use of low-dose computed tomography to assess pulmonary tuberculosis among healthcare workers in a tuberculosis hospital. Infect Dis Poverty. 2017 Mar 24;6(1):68

20. Kienzl-Palma D, Prosch H. Thoracic manifestation of tuberculosis. Radiologe. 2016 Oct;56(10):866873

21. McGuinness G, Naidich DP, Jagirdar J, Leitman B, McCauley DI. High resolution CT findings in miliary lung disease. J Comput Assist Tomogr 1992; 16:384-390

22. Chu H, Li B, Zhao L, Huang D, Xu J, Zhang J, Gui T, Xu L, Luo L, Zhang Z, Sun X. Tree-in bud pattern of chest CT images for diagnosis of Mycobacterium abscesses. Int J Clin Exp Med. 2015 Oct $15 ; 8(10): 18705-12$

23. Schiff HF, Jones S, Achaiah A, Pereira A, Stait G, Green B. Clinical relevance of non-tuberculous mycobacteria isolated from respiratory specimens: seven year experience in a UK hospital. Sci Rep. 2019 Feb 11;9(1):1730 
24. Kwak N, Lee CH, Lee HJ, Kang YA, Lee JH, Han SK, Yim JJ. Non-tuberculous mycobacterial lung disease: diagnosis based on computed tomography of the chest. Eur Radiol. 2016 Dec;26(12):44494456

25. Yasin H, Mangano WE, Malhotra P, Farooq A, Mohamed H. Hot Tub Lung: A Diagnostic Challenge. Cureus. 2017 Aug 27;9(8):e1617

26. Kim C, Park SH, Oh SY, Kim SS, Jo KW, Shim TS, Kim MY. Comparison of chest CT findings in nontuberculous mycobacterial diseases vs. Mycobacterium tuberculosis lung disease in HIV-negative patients with cavities. PLoS One. 2017 Mar 27;12(3):e0174240

27. Yuan MK, Chang CY, Tsai PH, Lee YM, Huang JW, Chang SC. Comparative chest computed tomography findings of non-tuberculous mycobacterial lung diseases and pulmonary tuberculosis in patients with acid fast bacilli smear-positive sputum. BMC Pulm Med. 2014 Apr 22;14:65

28. Kuhlman JE, Deutsch JH, Fishman EK, Siegelman SS. CT features of thoracic mycobacterial disease. RadioGraphics 1990; 10:413-431

29. Uitenbroek, D. G. (1997). SISA Exact test, https://www.quantitativeskills.com/sisa/statistics/fiveby2.htm

30. Ko JM, Park HJ, Kim CH. Clinicoradiologic evidence of pulmonary lymphatic spread in adult patients with tuberculosis. AJR Am J Roentgenol. 2015 Jan;204(1):38-43

31. Mehta AR, Mehta PR, Mehta RL. A cough conundrum in a patient with a previous history of BCG immunotherapy for bladder cancer. BMJ Case Rep. 2012 Oct 24;2012

32. Marusić A, Katalinić-Janković V, Popović-Grle S, Janković M, Mazuranić I, Puljić I, Sertić Milić H. Mycobacterium xenopi pulmonary disease - epidemiology and clinical features in nonimmunocompromised patients. J Infect. 2009 Feb;58(2):108-12 


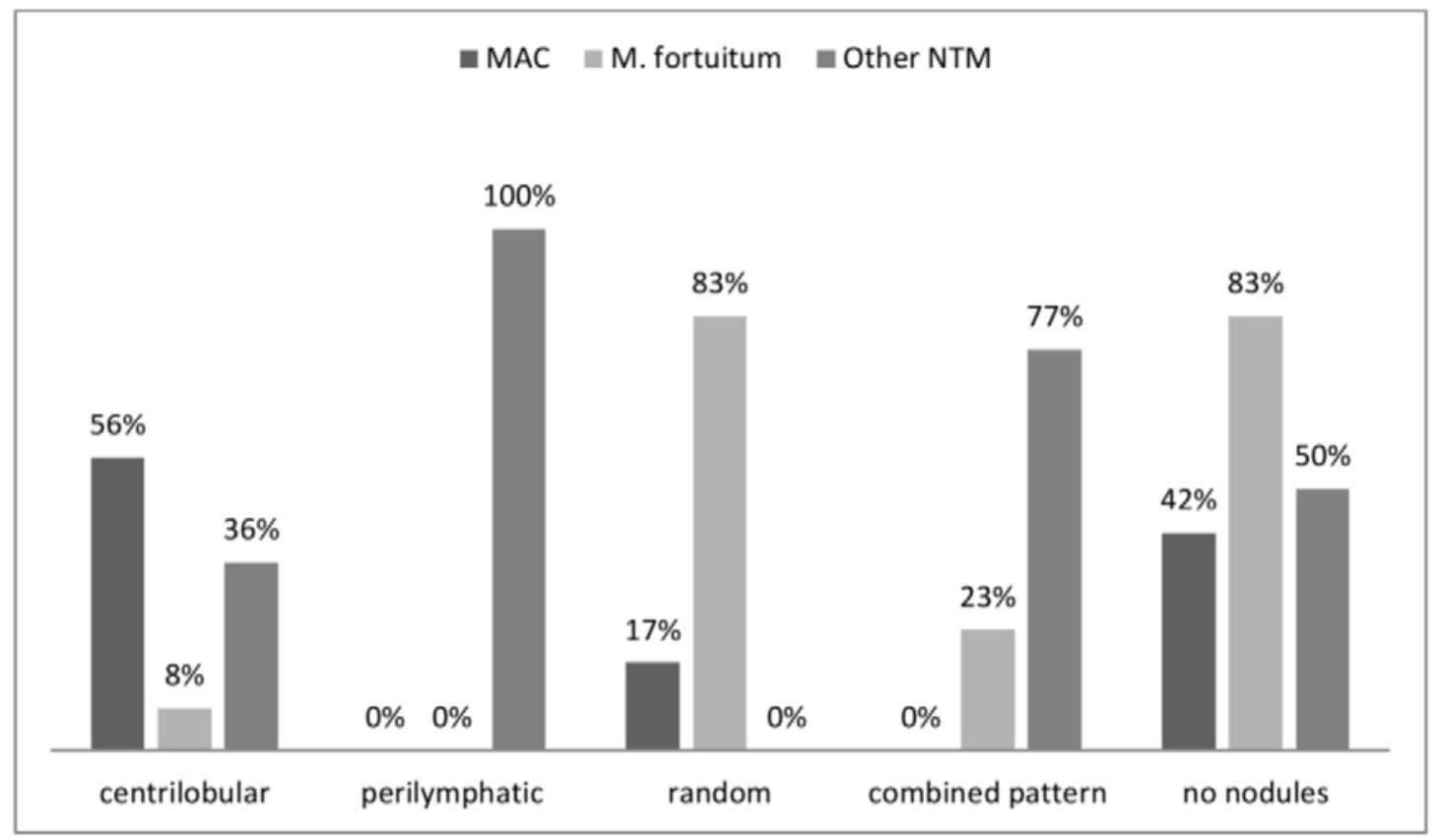

Figure 1 The proportion of NTM species in the created group (centrilobular, perilymphatic, random, combined pattern and no nodules group).

* MAC: patients with identified M. avium and M. intracellulare complex, Other NTM: patients with identified M. abscessus, M. chimerae, M. malmoense, M. celatum, M. kansasii or M. segmatis 

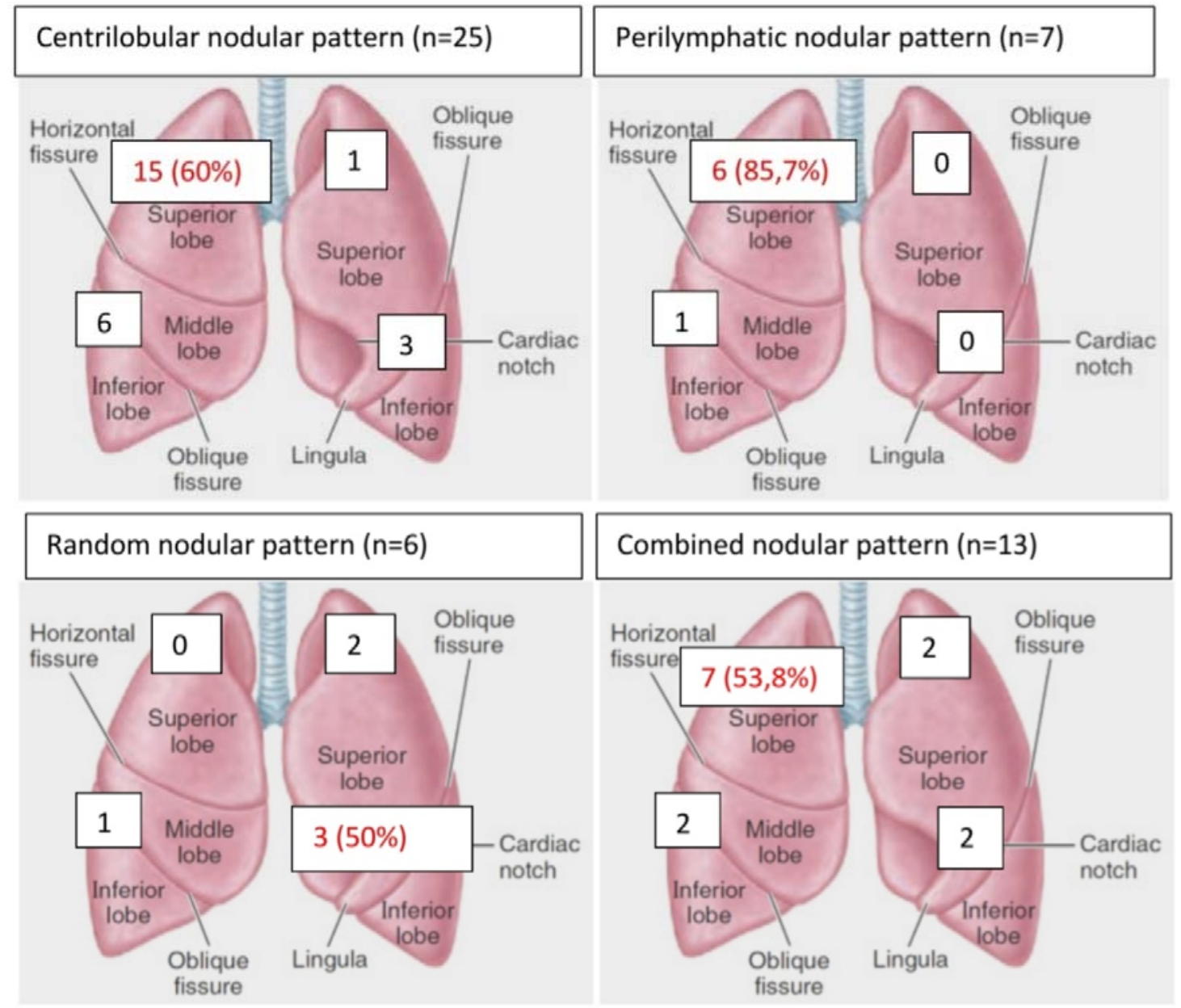

Figure 2 Distribution of nodule pattern in the lung. 

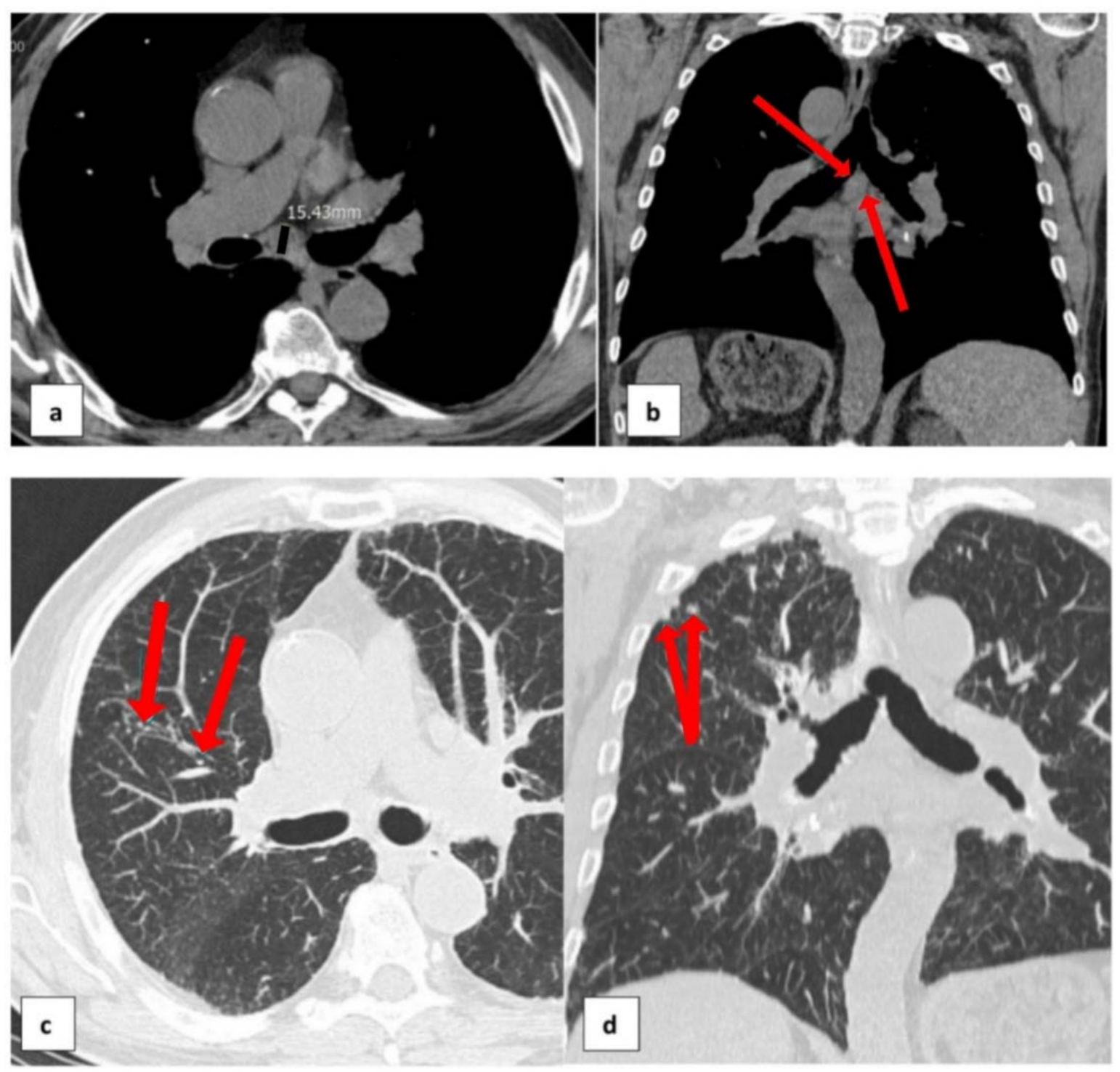

Figure 3 The same patient.

a) and b) chest $\mathrm{CT}$ - the axial and coronal cross sections show subcarinal lymphadenopathy.

c) and d) chest CT - lung window, $5 \mathrm{~mm}$ Maximum Intensity Projection (MIP) - the axial and coronal cross sections show perilymphatic nodule distribution pattern with right and upper side predominance in the lung. 